\title{
Solving Linear Recurrence Equations
}

\author{
Yongjae Cha \\ Mark van Hoeij* \\ Giles Levy \\ ycha@math.fsu.edu hoeij@math.fsu.edu glevy@math.fsu.edu
}

\author{
Department of Mathematics \\ Florida State University \\ Tallahassee, FL 32306, USA
}

The software to be presented is an implementation of the algorithms in [1], [2], and [3]. (This software is available at [4].) The main algorithm in [3] is currently implemented with additional base equations beyond what appear in [3] and will be part of the software demonstration in July.

Common to each algorithm is a transformation from a base equation to the input using transformations that preserve order and homogeneity (referred to as gt-transformations). Before calling any of these algorithms we first check currently available algorithms for a solution in the form of a first order right hand factor or some other general term solution.

Algorithm 'Find ${ }_{2} F_{1}$ ' will find a gt-transformation to a recurrence relation satisfied by a hypergeometric series $u(n)={ }_{2} F_{1}\left({ }^{a+n}{ }_{c} b \mid z\right)$, if such a transformation exists. As an example, sequence $A 005572=[1,4,17,76,354,1704,8421, \ldots]$ from the OEIS ([5]) represents the "Number of walks on cubic lattice starting and finishing on the xy plane and never going below it." $A 005572$ has offset 0 (i.e. the first entry in the list is $A 005572(0)$ ) and satisfies:

$$
12(n+1) A 005572(n)-(20+8 n) A 005572(n+1)+(n+4) A 005572(n+2)=0 .
$$

The output from our program is: $A 005572(n)=$

$$
\frac{2^{n+1}}{\sqrt{3}(n+2)}(2 \cdot \operatorname{hypergeom}([1 / 2, n+2],[1], 2 / 3)-3 \cdot \operatorname{hypergeom}([1 / 2, n+1],[1], 2 / 3)), n \geqslant 0 \text {. }
$$

The algorithm 'Find Liouvillian' will find a gt-transformation to a recurrence relation of the form $u(n+2)+b(n) u(n)=0$ for some $b(n) \in \mathbb{C}(n)$, if such a transformation exists. 'Find Liouvillian' is not unique in terms of its purpose but, for second order recurrence relations, it is faster than prior algorithms. As an example, for the recurrence relation satisfied by A099364 from the OEIS, our program produces the solution:

$$
\begin{gathered}
A 099364(n)=\left(\frac{1}{6} n+\frac{5}{6}\right) v(n)-\left(\frac{1}{12} n+\frac{1}{2}\right) v(n+1), \\
\text { where } v(n+2)-\frac{4(n+2)}{n+7} v(n)=0 .
\end{gathered}
$$

The algorithm 'Database Solver' takes advantage of a large database of sequences, the OEIS mentioned above, by using the recurrence relations that they satisfy as base equations. We have already partitioned the database into subsets such that there exists a gt-transformation between any two members of the same subset. For each subset there exist invariant properties shared by all members which we compare to the invariant properties of the input to narrow the search for

${ }^{*}$ Supported by NSF grant 0728853 
a relationship. If the input is a recurrence relation with initial conditions defining a sequence then the output will be a transformation from a representative sequence (if there is a match in the database). The output could be useful since the representatives are chosen, in large part, for how much information there is about that sequence (formulas, papers citing the sequence, ...).

As an example of this algorithm, suppose we were working with the "Number of $n X n$ symmetric $(0,1)$-matrices with exactly $n+1$ entries equal to 1 and no zero rows or columns" (sequence $A 135593$ from the OEIS). This sequence, $[2,9,36,140,540,2142, \ldots]$ has offset 2 and satisfies:

$n(n-3) A 135593(n+2)-(n+2)(n-4) A 135593(n+1)-(n-2)(n+2)(n+1) A 135593(n)=0$.

We enter the following into our program:

$$
n(n-3) u(n+2)-(n+2)(n-4) u(n+1)-(n-2)(n+2)(n+1) u(n), u(n),\{u(2)=2, u(3)=9\}
$$

and the following output is returned:

$$
u(n)=A 000085(n)+\frac{n-2}{2} A 000085(n+1), n \geqslant 2 .
$$

There is much information in the form of formulas, comments, references, cross-references to other sequences, and world wide web URL links on A000085's page and not much (currently) on the page for $A 135593$. A000085 is the "number of self-inverse permutations on $\mathrm{n}$ letters, also known as involutions" and just as interesting are the many additional definitions, from the comments, such as "the number of graphs on $\mathrm{n}$ labeled nodes with degrees at most 1 " and "the number of partitions of a set of n distinguishable elements into sets of size 1 and 2."

By computing local data of a recurrence equation (see [3] for details) the program can find gt-transformations to many different known functions. Currently in our table of known functions are Bessel (first and second kind), Whittaker (W and M), and types of Jacobi, Legendre, Laguerre, and Gegenbauer functions. As an example, for the recurrence equation satisfied by the interesting sequence $A 143415$ from the OEIS, the program will return the solution:

$$
A 143415(n)=\frac{1}{2} \sqrt{\frac{e}{\pi}}\left(\frac{\operatorname{BesselK}\left(n-\frac{1}{2}, \frac{1}{2}\right)+(2 n-1) \operatorname{BesselK}\left(n+\frac{1}{2}, \frac{1}{2}\right)}{n(n+1)}\right), n \geqslant 1 .
$$

\section{References}

[1] G. Levy. Solutions of second order recurrence relations. Ph.D. dissertation, Florida State University, 2010. Text and implementations available at: http://www.math.fsu.edu/ glevy/

[2] M. van Hoeij, G. Levy. Liouvillian Solutions of Irreducible Second Order Linear Difference Equations. To appear in ISSAC '10: Proceedings of the 2010 International Symposium on Symbolic and Algebraic Computation, 2010.

[3] Y. Cha, M. van Hoeij, G.Levy. Solving Recurrence Relations using Local Invariants. To appear in ISSAC '10: Proceedings of the 2010 International Symposium on Symbolic and Algebraic Computation, 2010.

[4] Implementation. http://www.math.fsu.edu/ glevy/implementation/code.zip

[5] N. J. A. Sloane. The On-Line Encyclopedia of Integer Sequences. World-Wide Web URL www.research.att.com/ njas/sequences/ 